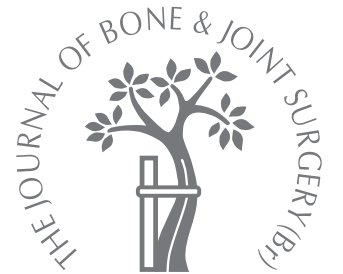

W. C. Hembree,

B. D. Ward,

B. D. Furman,

R. D. Zura,

L. A. Nichols,

F. Guilak,

S. A. Olson

From Duke

University Medical

Center, Durham,

USA

\title{
Viability and apoptosis of human chondrocytes in osteochondral fragments following joint trauma
}

\begin{abstract}
Post-traumatic arthritis is a frequent consequence of articular fracture. The mechanisms leading to its development after such injuries have not been clearly delineated. A potential contributing factor is decreased viability of the articular chondrocytes. The object of this study was to characterise the regional variation in the viability of chondrocytes following joint trauma. A total of $\mathbf{2 9}$ osteochondral fragments from traumatic injuries to joints that could not be used in articular reconstruction were analysed for cell viability using the fluorescence live/dead assay and for apoptosis employing the TUNEL assay, and compared with cadaver control fragments.

Chondrocyte death and apoptosis were significantly greater along the edge of the fracture and in the superficial zone of the osteochondral fragments. The middle and deep zones demonstrated significantly higher viability of the chondrocytes. These findings indicate the presence of both necrotic and apoptotic chondrocytes after joint injury and may provide further insight into the role of chondrocyte death in post-traumatic arthritis.
\end{abstract}

Post-traumatic arthritis (PTA) is a common consequence of displaced articular fractures. It is estimated that as much as $10 \%$ of symptomatic osteoarthritis (OA) in the general population can be classified as post-traumatic. ${ }^{1}$ The mechanisms leading to the development of PTA after articular fractures have not been clearly delineated, but one of the potential contributing factors is decreased viability of the articular chondrocytes. It is not possible to critically assess their viability in situ in patients following articular fractures, but small osteochondral fragments from the zone of injury which cannot be used in articular reconstruction can be assessed for viability. Previous reports ${ }^{2,3}$ have noted a generalised decrease in chondrocyte viability or increased rates of apoptosis in such fragments. However, none has critically evaluated the regional variation of viability within the cartilage layer.

The aim of this study was to assess regional variations in the viability of chondrocytes in osteochondral fragments resulting from traumatic disruption of a joint. We wished to determine how the viability of the cells varies within the articular cartilage at locations both close to and away from the edge of a fracture. We also investigated how viability of the cells correlated with the type of joint, the age of the patient and the time from injury. We hypothesised that chondrocytes located along the frac- tured edges of fragments of cartilage would demonstrate significantly decreased viability compared to those located away from this site.

\section{Materials and Methods}

A total of 29 osteochondral fragments were collected at the time of surgery from patients undergoing open reduction and internal fixation of a traumatic joint injury in accordance with protocols approved by the Institutional Review Board of Duke University Medical Center. The samples were not used in reconstruction of the joint and would otherwise have been discarded. In all cases, the specimens had the gross appearance of a full-thickness layer of articular cartilage that remained in continuity with a fragment of subchondral bone. The transit time from the operating room to the laboratory was approximately 15 minutes. The only information recorded concerning the specimens was the age of the patient ( $>89$ years recorded as $90+$ ), the gender, and the time since the injury $(\leq 24$ hours recorded as one day). Given the wide array of severity of injury, it was not always possible to specify the exact anatomical source of each fragment, so only the joint from which the fragment was obtained was recorded. The fragments were stored at $4^{\circ} \mathrm{C}$ in either normal saline or phosphate-buffered saline (PBS) until further processing. In all cases, the cartilage 


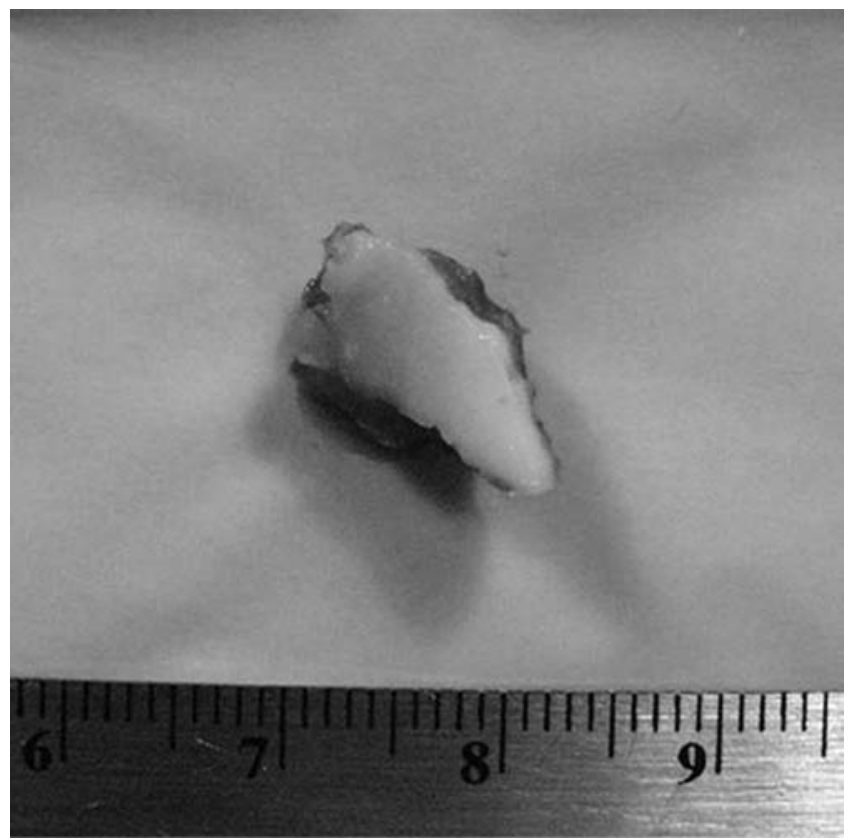

Fig. 1a

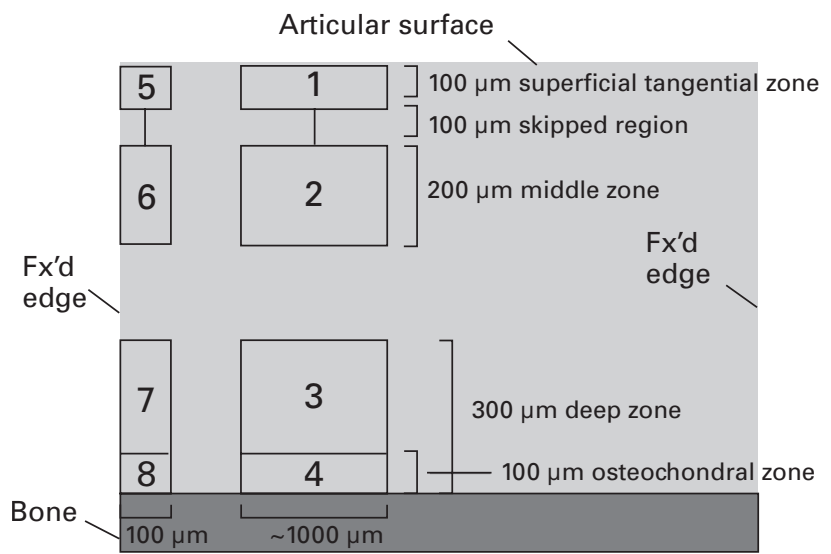

Fig. 1c

layer was left in continuity with the subchondral bone during processing, which was begun within 24 hours of collection. Prior to processing, a gross digital photograph was taken of all samples and their scale was determined by placing a ruler in each photograph (Fig. 1a). The surface area $\left(\mathrm{mm}^{2}\right)$ was later calculated for each sample using image analysis software (Zeiss, Thornwood, New York) (Fig. 1b).

Live-dead assay for chondrocyte viability. The cell viability of each osteochondral sample was assessed using a live/ dead assay (Invitrogen Molecular Probes, Carlsbad, California). Each sample was halved in a plane orthogonal to the articular surface (Fig. 1c). One of the halves was reserved for the terminal deoxynucleotidyl transferase nick end labelling (TUNEL) assay and the other was washed in PBS for five minutes and then labelled with a

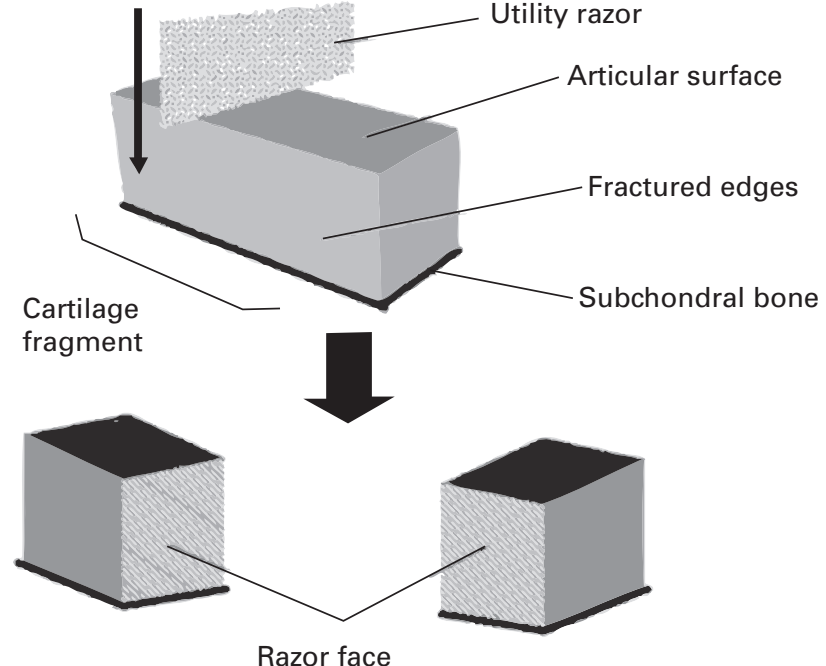

Fig. 1b

Analysis and preparation of samples. a) Samples were collected and a digital photograph was taken. b) Samples were then halved in a plane orthogonal to the articular surface. c) Zones of interest were delineated on the razor face of the sample. Zones 1 to 4 represent the central region. Zones 5 to 8 represent the fractured edge. Fx'd, fractured edge. Diagram not to scale.

live/dead assay ( $3 \mu \mathrm{l}$ calcein $\mathrm{AM}$ and $3-\mu \mathrm{l}$ ethidium homodimer-1 dissolved in $3 \mathrm{ml}$ of PBS). The sample and solution were wrapped in aluminium foil to prevent exposure to light, and incubated at room temperature for 25 minutes. After labelling, the sample was washed twice in PBS for five minutes and then placed face down in a chambered cover glass. A confocal laser scanning microscope (LSM (510; Zeiss) was used to view the sample (objective $10 \times$, numerical aperture 0.30 ). Because the diameter of a human chondrocyte is approximately $12 \mu \mathrm{m}$ to $13 \mu \mathrm{m}$, an optical slice of $15 \mu \mathrm{m}$ was chosen in an effort to capture fluoroscence from only one cell layer within the tissue. Images were recorded at greater than $50 \mu \mathrm{m}$ into the face of the sample to ensure that cells from the cut edge of the specimens were eliminated. Six images were taken from 


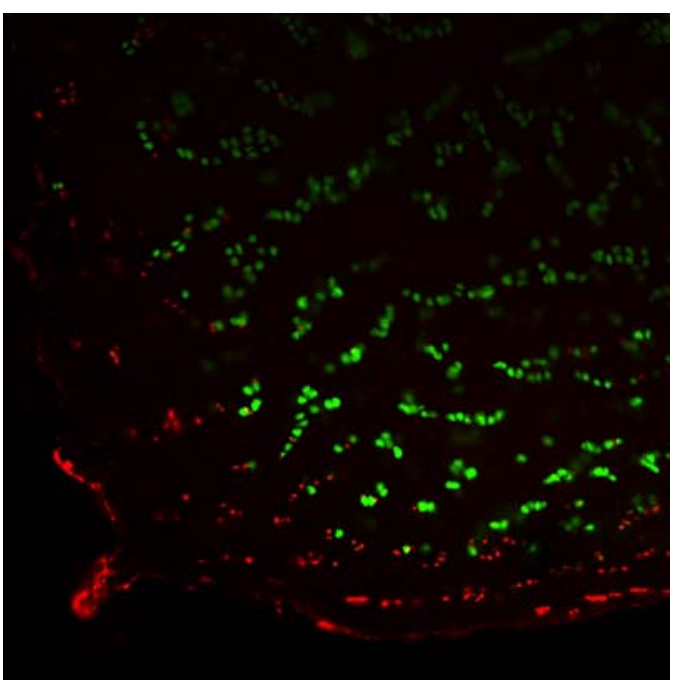

Fig. 2a

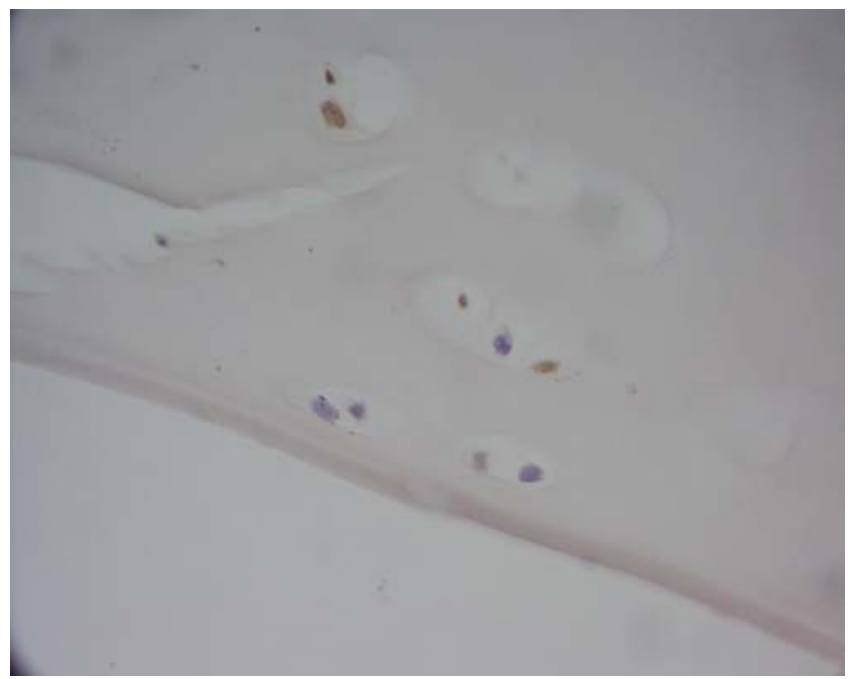

Fig. $2 b$

Live/dead and terminal deoxynucleotidyl transferase nick end labelling (TUNEL) assay images. a) Representative confocal microscope image from the live/dead assay of the superficial and middle zones of the fractured region. Live cells stain green with the calcein stain, whereas dead cells stain red with ethidium. b) Photomicrograph of the superficial zone in the fractured region after TUNEL staining. Apoptotic chondrocyte nuclei stain brown and viable chondrocyte nuclei stain blue with haematoxylin (400× original magnification).

each sample, three away from the fracture edge (central region) and three along the edge. After the images had been recorded and saved, the samples were fixed in $10 \%$ buffered formaldehyde and stored at $4{ }^{\circ} \mathrm{C}$ for histological analysis.

Two regions of interest were chosen for image analysis, one located centrally away from the edge of the fracture and one along the edge (Fig. 1). Because the cellular and structural components of articular cartilage vary according to depth, each region was further divided into four dis-

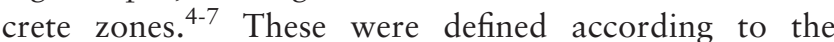
structure of the cartilage and included the superficial, middle, deep, and osteochondral zones. Laser scanning microscope software (Zeiss) was used to delineate each zone of interest on the appropriate images captured from each sample (Fig. 2). The superficial zone was defined as the first $100 \mu \mathrm{m}$ of tissue from the articular surface, so as to ensure that the zone lay within the top $10 \%$ of the articular cartilage regardless of the total thickness of the fragment. The middle zone was defined as the tissue located between $200 \mu \mathrm{m}$ and $400 \mu \mathrm{m}$ from the articular surface, and comprised $60 \%$ of the cartilage. The deep zone was defined as the cartilaginous tissue extending $300 \mu \mathrm{m}$ inward from the tidemark, the bottom $30 \%$ of cartilage. The osteochondral zone was defined as the cartilaginous tissue extending $100 \mu \mathrm{m}$ inward from the tidemark. This was a subset of the deep zone and was chosen to examine the cartilage within $10 \%$ of the osteochondral interface.

For the central region, the width of each zone was equal to the width of the image, approximately $1000 \mu \mathrm{m}$. For the region of the edge of the fracture, the width of each zone was set at $100 \mu \mathrm{m}$ from the edge in order to ensure that the zones along the edge included tissue closest to the fracture. The area of each delineated zone was recorded $\left(\mu \mathrm{m}^{2}\right)$. By defining the zones in this manner, the effect of variation of the thickness of the cartilage between samples was eliminated.

After delineating the zones of interest on the appropriate images, the dead cells, as shown by red fluorescence, and the live cells, with green fluorescence, in each image, were exported separately. The images were then imported into an image analysis package (Scion Image; Scion Inc., Frederick, Maryland). For all images, intensity thresholds of 65 and 80 (0 to 255 scale) were selected to identify dead and live cells, respectively. All images were converted to binary images and eroded once to better define the individual cells. Each previously defined zone was analysed separately. All cells within that zone were then counted using a minimum particle size of two for dead and five for live. Using these data, the number of total cells in each zone of interest was determined [total cell number $=$ live cell number + dead cell number] and used to calculate the percent viability of cells $[\%$ viability $=($ live cell number $/$ total cell number) $\times 100]$.

TUNEL assay for chondrocyte apoptosis. Apoptotic cells were labelled in situ using a commercial TUNEL assay kit (ApopTag Peroxidase In Situ Apoptosis Detection Kit; Chemicon International, Temecula, California). Following fixation in $10 \%$ neutral buffered formalin, all the samples were decalcified, dehydrated, embedded in paraffin, and sectioned perpendicular to the articular surface at a thickness of $5 \mu \mathrm{m}$. 
Tissue sections were deparaffinised and incubated in proteinase $\mathrm{K}(40 \mu \mathrm{g} / \mathrm{ml})$ for 25 minutes. Endogenous peroxidase was then quenched by incubation in $3.0 \%$ hydrogen peroxide for five minutes. The ends of fragmented DNA in the tissue were labelled with terminal deoxynucleotidyl transferase $(\mathrm{TdT})$ in the presence of digoxigenin-conjugated nucleotides and unlabelled nucleotides for 75 minutes at $37^{\circ} \mathrm{C}$. The samples were then incubated for 30 minutes with anti-digoxigenin antibodies that were conjugated to a peroxidase reporter molecule. Immunohistochemical detection of these antibodies was carried out by exposure to a chromogenic substrate for 15 minutes (ImmunoPure, Metal Enhanced DAB Substrate; Pierce, Rockford, Illinois). All slides were then counterstained with Harris' Haematoxylin (Poly Scientific, Bay Shore, New York). This was followed by dehydration in alcohol, a xylene rinse, and mounting under a coverglass.

Light microscopy was used to determine the percentage of apoptotic cells. Brown nuclei indicated apoptotic cells and blue nuclei the viable cells (Fig. 2). Two tissue sections from each sample were analysed. Identical zones and regions to those described above were used. The superficial, middle, deep and osteochondral zones were delineated using a reticle for both the edge of the fracture and the central region. The number of apoptotic and viable cells in each zone of interest was counted three times, and then averaged. Using these data, the number of total cells in each zone of interest was determined [total cell number $=$ apoptotic cell number + viable cell number] and used to calculate the percentage of apoptotic cells $[\%$ apoptosis $=($ apoptotic cell number/total cell number $)$ $\times 100]$.

Control samples. Human knee and ankle joints received within 24 hours of death were used as controls. Cartilage explants were harvested from these specimens using a $6 \mathrm{~mm}$ dermal biopsy punch. The viability and apoptosis of the cells were assessed in the explants using protocols identical to those described above.

Two positive controls were used for TUNEL analysis. The first was made by subjecting fresh porcine explants to 24 hours of incubation in Ham's F12 culture media with $4 \mathrm{mM}$ of $\mathrm{H}_{2} \mathrm{O}_{2}$ to induce apoptosis. The second was a commercially-available slide of a tissue section from rat mammary glands (ApopTag Positive Control Slides; Chemicon International).

Statistical analysis. Statistical analysis was performed using multifactorial analysis of variance (ANOVA) with significance reported at the $95 \%$ confidence level. The time after injury was not normally distributed and was therefore grouped as follows: zero to one day, one to two days, two to 10 days, > 10 days, and treated as a categorical variable. Regression analysis was used to analyse the relationship between the age of the patient, the twodimensional (2D) surface area of the fragment, and the percentage viability of the chondrocytes in each zone.
Table I. Summary (mean, range) of age, time status post (s/p) trauma, and surface area of samples used for live/dead and apoptosis studies

\begin{tabular}{lcr}
\hline & Live/dead assay & TUNEL $^{*}$ assay \\
\hline Age (yrs) & 43 (19 to 77$)$ & 42 (19 to 77$)$ \\
Time s/p trauma (days) & 5 (1 to 24$)$ & 5 (1 to 24$)$ \\
Surface area $\left(\mathrm{mm}^{2}\right)$ & 90 (15 to 268$)$ & 87 (15 to 268) \\
\hline
\end{tabular}

* TUNEL, terminal deoxynucleotidyl transferase nick end labelling

Table II. Summary data of specimens. Anatomic location and number of specimens used for each analysis

\begin{tabular}{lll}
\hline Anatomic location & Live/dead assay & $\begin{array}{l}\text { TUNEL }^{*} \\
\text { assay }\end{array}$ \\
\hline Knee & 10 & 7 \\
Hip & 9 & 7 \\
Ankle & 6 & 6 \\
Elbow & 3 & 3 \\
Foot & 1 & 1 \\
\hline * TUNEL, terminal deoxynucleotidyl transferase nick end \\
labelling
\end{tabular}

\section{Results}

Samples received, age, anatomical location, time from injury. A total of 29 usable samples were obtained for the live/dead assay and subsequent analysis. A TUNEL analysis was performed on 24 of these samples. Five could not be used because of inadequate histological preparation or poor nuclear staining in the TUNEL assay. The characteristics of the samples are summarised in Tables I and II.

Chondrocyte viability. In the central region, the superficial zone demonstrated an average viability of $42.1 \%$, which was less than all other zones in the region (Fig. 3). The middle, deep and osteochondral zones showed an average viability of $84.2 \%, 74.5 \%$ and $60.0 \%$, respectively. Along the edge of the fracture, the superficial zone demonstrated an average viability of $24.1 \%$, which was less than in all other zones. The middle, deep and osteochondral zones had an average viability of $37.6 \%, 43.6 \%$ and $36.5 \%$, respectively. The viability of cells within the superficial zone was significantly less (ANOVA, $\mathrm{p}<0.05$ ) than in the middle and deep zones, regardless of region. The osteochondral zone also demonstrated decreased viability relative to the middle and deep zones, but the difference was not significant. The edge of the fracture demonstrated significantly less (ANOVA, $\mathrm{p}<0.05$ ) viability than the central region across all zones (Fig. 3).

Chondrocyte apoptosis. In the central region, the superficial zone demonstrated an average of $32.2 \%$ apoptotic cells, which was higher than all other zones in the region (Fig. 4). The middle, deep and osteochondral zones demonstrated an average of $20.3 \%, 20.1 \%$ and $21.6 \%$, respectively. Along the edge of the fracture, the superficial 


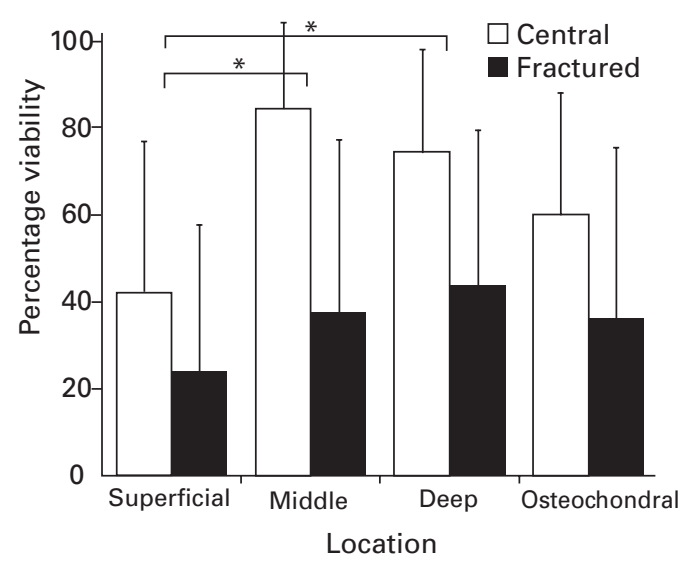

Fig. 3

Cell viability in osteochondral fragments as measured using a fluorescent live/dead assay. The superficial tangential zone demonstrates an average percentage viability that is significantly less than the middle and deep zones, regardless of region. The fractured edge demonstrates an average percentage viability that is significantly less than the central region across all zones. The error bars indicate mean and standard deviation ( ${ }^{*}$ ANOVA, $\mathrm{p}<0.05$ ).

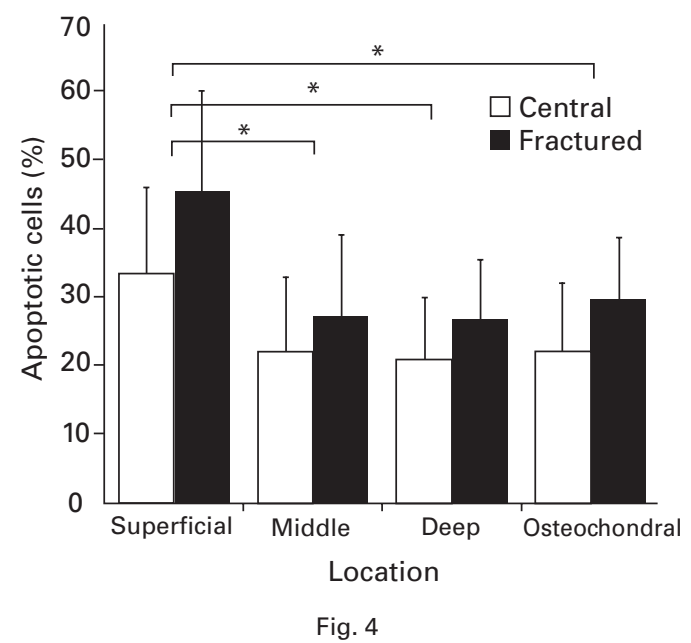

Cell apoptosis as measured by TUNEL assay. The superficial zone demonstrated a significantly greater percentage of apoptotic cells than the middle, deep and osteochondral zones, regardless of region. The fractured edge demonstrated a greater percentage of apoptotic cells than the central region across all zones. The error bars indicate mean and standard deviation ( ${ }^{*}$ ANOVA, $p<0.05$ ). zone showed an average of $42.9 \%$ apoptotic cells, which was higher than in all other zones. The middle, deep and osteochondral zones had $25.7 \%, 25.8 \%$ and $28.9 \%$ of apoptotic cells, respectively. Similar to the results of the live/dead assay, the superficial zone demonstrated a significantly higher percentage of chondrocyte apoptosis (ANOVA, p < 0.05) than the middle, deep and osteochondral zones, regardless of region. The edge of the fracture also had a greater percentage of apoptotic cells (ANOVA, $\mathrm{p}<0.05$ ) than the central region across all zones (Fig. 4). Control samples. The specimens of cartilage from fresh human cadaver tissue demonstrated an average viability across all zones of approximately $97.5 \%$, as measured with the live/dead assay, and an average of $3.5 \%$ apoptotic cells across all zones as measured with the TUNEL assay.

Anatomical location, age, size of fragment, and time since injury. Analyses of the anatomical location, the age of the patient, the size of the fragment and time since injury were performed separately for cell viability and apoptosis. Multifactorial ANOVA revealed no significant difference in cell viability or apoptosis with anatomical location across all zones (ANOVA, $\mathrm{p}>0.05$ ). Regression analysis revealed no correlation between the age of the patient and either percentage viability or apoptosis of the cells (linear regression; $\mathrm{r}^{2}<0.1, \mathrm{p}>0.05$ ). No correlation was observed between the $2 \mathrm{D}$ surface area of the fragment and cell viability or apoptosis (linear regression; $\left.\mathrm{r}^{2}<0.1, \mathrm{p}>0.05\right)$. There was no significant difference in cell viability with the time since injury (Fig. 5), but the TUNEL assay revealed a dependence on this factor with apoptosis. Samples collected between one and two days after injury showed the highest percentage of apoptotic cells (Fig. 5). This difference was significant compared to samples collected between two and 10 days after fracture (ANOVA, $\mathrm{p}<0.05$ ).

\section{Discussion}

The findings of this study support the hypothesis that traumatic fracture of an articular surface results in significant chondrocyte death and apoptosis, with cells along the edges of the disrupted articular cartilage showing decreased viability and increased apoptosis compared to those located away from this site. The articular surface of the cartilage also demonstrates a marked decrease in chondrocyte viability, as measured with both the live/dead assay and the TUNEL assay, regardless of whether it is located along the edge of the fracture or away from it. The middle and deep zones of cartilage away from the fracture appear to be privileged areas, with greater cell viability than other zones. Although the live/dead assay demonstrated no correlation between time from injury and cell viability, the TUNEL assay showed a small but significant increase in apoptotic cells one to two days after injury. No correlation was observed between cell viability and the age of the patient.

There were certain limitations to this study, arising mainly from the constraints of collecting only discarded samples from the operating theatre. Nonetheless, our findings showed a consistent trend when averaged among different joints, suggesting that similar mechanisms may be involved in cell death after traumatic articular fracture. 

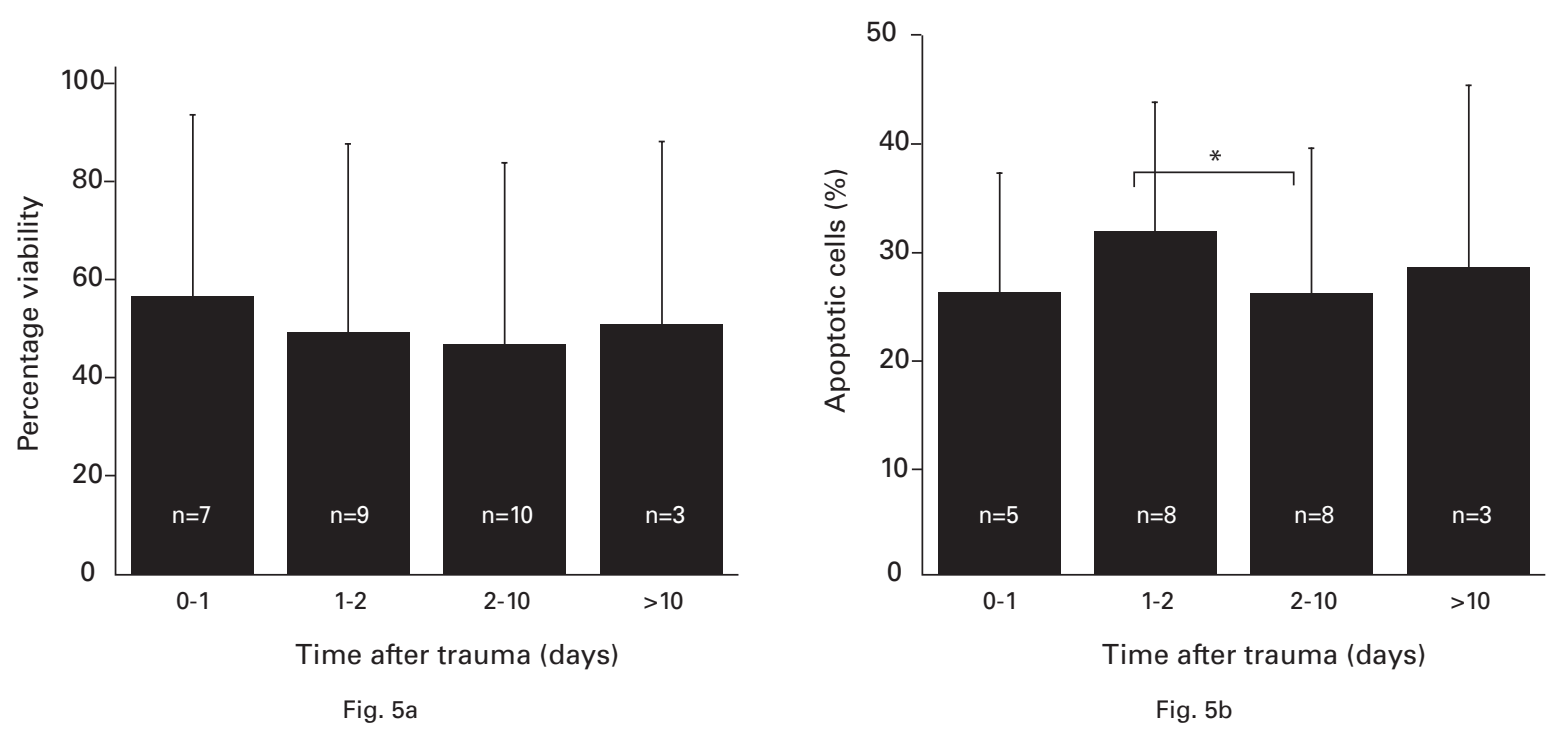

Graphs showing the effects of time after trauma on cell viability and apoptosis. a) Percentage viability by live/dead assay demonstrated no difference with time following injury, and b) percentage apoptotic cells by terminal deoxynucleotidyl transferase nick end labelling assay (TUNEL) demonstrated a significantly higher fracture of apoptotic cells at one to two days than after two to 10 days. The error bars indicate mean and standard deviation ("ANOVA, $p<0.05$ ).

Chondrocyte death in osteochondral fragments was evaluated using two different methods: a live/dead assay using fluorescence confocal microscopy on fresh tissue, and an in situ TUNEL assay on fixed tissue. The live/dead assay identifies live cells through the activity of intracellular esterases that induce fluorescence of the calceinAM molecule, whereas dead cells are identified by the ability of the ethidium homodimer to penetrate a compromised cell membrane and fluorescently label the cell nucleus. It is important to note that in the early stages of apoptosis, cells are still viable and may exhibit positive labelling with calcein-AM, so there is some potential for overlap between apoptotic and live cells, as determined using the live/dead assay and TUNEL staining.

The fluorescent live/dead assay showed significantly higher cell death in the cartilage surface as well as the fractured edge of the fragments. The decreased viability in the superficial zone is consistent with studies of in vitro impact to the articular surface in explant models of traumatic injury. ${ }^{8-10}$ The mechanism resulting in higher cell death at the tissue surface may involve a combination of factors that result in higher levels of cell and tissue strains at the tissue surface. Articular cartilage exhibits inhomogeneity of mechanical properties in tissues within the different zones. In particular, several studies have shown that the superficial zone has a significantly lower compressive modulus than the middle or deep zones, ${ }^{11-13}$ and thus chondrocytes in the superficial zone undergo a higher magnitude of stress and strain than those in the middle or deep zones during tissue compression. ${ }^{14-16}$ Theoretical models of cartilage impact suggest that even if the mechanical properties of tissue were uniform with depth, the stress-strain environment within the cartilage is predicted to be inhomogeneous, with the highest shear stresses at the tissue surface and at the interface with subchondral bone. ${ }^{17,18}$

Other studies have shown that the extent and distribution of cell death in cartilage are dependent on the rate and orientation of joint loading. ${ }^{19,20}$ Although the cause of injury was not recorded in our study, the mechanisms generally involve relatively high rates of impact resulting from motor vehicle collisions, falls, or acts of violence. These types of injury can involve acute impact to the cartilage surface, either by a foreign object or by a bone-on-bone mechanism. Such acute impacts are consistent with the reduced cell viability observed in the superficial zones of our samples. However, some of the variability in our findings is attributable to the various potential mechanisms of joint injury and articular fracture.

The decreased viability observed along the edges of the fracture of osteochondral fragments was not unexpected and probably occurred because of damage to the cartilage matrix and macromolecular structure as the tissue was fractured during impact and injury. The fracture strain of cartilage is relatively high compared to that of bone, and in adult cartilage may be as high as $60 \% .^{21}$ In other studies, it has been shown that such magnitudes of strain are sufficient to cause chondrocyte death under quasi-static loading conditions. ${ }^{15,22}$ Also, chondrocytes at the tissue edges are likely to be exposed to a higher concentration of blood products and pro-inflammatory cytokines within 
the joint space than the cells that are more distant from the fracture. Recent studies suggest that the presence of leucocytes and their associated production of proinflammatory mediators may be partly responsible for chondrocyte death following impact. ${ }^{23}$

The osteochondral zone located away from the site of fracture demonstrated a trend towards decreased viability compared to the middle and deep zones in that region, although this finding was not statistically significant as measured with the live/dead assay. Earlier studies in animals have demonstrated that the osteochondral interface is subject to damage and cell death during articular impact even when other zones of the cartilage are left intact. ${ }^{24-26}$ This characteristic is consistent with the presence of high shear stresses at the interface during impact, because of the difference in mechanical properties between the cartilage and subchondral bone. ${ }^{17,18}$

Significant chondrocyte apoptosis was detected using the TUNEL assay in the osteochondral fragments compared to the control specimens. This finding is consistent with a previous study by Murray et $\mathrm{al}^{2}$ showing that, on average, $35 \%$ of chondrocytes were TUNEL-positive in osteochondral fragments similar to those used in the present study. Similarly, studies of cartilage explants have reported measurements of both apoptotic and necrotic cells associated with impact loading and cartilage defects. ${ }^{11,27}$ An important observation of our study was the time course of cell necrosis and apoptosis as measured with the live/dead assay and the TUNEL assay, respectively. Previous studies that have looked at the effects of impact loading on cell viability have done so by investigating apoptosis and applying the general terminology of 'chondrocyte death' to that process. ${ }^{3,25,28}$ In a study on impact loading of bovine articular explants, Loening et $\mathrm{al}^{25}$ described a maximal state of apoptosis at 24 hours after impact loading. Other studies in humans have observed longer time periods. Kim et $\mathrm{al}^{3}$ found maximal apoptosis at five days after traumatic injury to human cartilage. Most of our samples were received between two and four days after injury (Table I), but some were analysed at more than seven days. The live/dead assay in our study demonstrated no correlation between the time after injury and cell viability. However, data obtained using the TUNEL assay demonstrated a small but significant peak in apoptosis one to two days after injury. The lack of correlation between the time after injury and cell viability as measured with the live/dead assay indicates that early surgery with adequate reduction may not necessarily protect some chondrocytes from dying. However, the fact that apoptosis increases several days after trauma raises questions as to when future novel therapies such as antiapoptotic agents, growth factors, and corticosteroids might best be applied to protect cells. Such therapies could prove valuable in the treatment of PTA.

The current literature suggests that an increase in chondrocyte apoptosis is associated with the cartilage damage and degeneration that is commonly seen in OA. The initial exposure of cartilage to blood at the time of injury can also induce chondrocyte apoptosis. ${ }^{29}$ The pro-inflammatory cytokine tumour necrosis factor- $\alpha$ has been reported to induce apoptosis in both hypertrophic and nonhypertrophic chondrocytes in a dose-dependent manner. ${ }^{30}$ Necrosis results in a corresponding inflammatory response that leads to the destruction of adjacent tissue and may influence both the catabolic and the anabolic metabolism of cartilage following joint injury. Further investigations are needed to improve our understanding of the role of necrosis and apoptosis after articular fracture, and how these distinct mechanisms of cell death contribute to the progression of PTA.

This study demonstrated no correlation between the age of the patient and the viability of cells across all eight zones of interest. Earlier studies have shown that age is a risk factor for PTA, and that, in general, older patients are at higher risk for its occurrence after traumatic joint injury. ${ }^{31-33}$ This might be attributable to the reduced response to injury of chondrocytes in older individuals. Studies have also shown that the chondrocytes of both younger humans and younger animals have a better proliferative response to injury and secrete larger matrix proteins after injury, ${ }^{34-36}$ suggesting that the chondrocytes of older patients might not be as capable of repairing damage to the cartilage matrix as are those of younger individuals. However, the results of our study provide evidence that age does have a significant effect on chondrocyte susceptibility to necrosis or apoptosis following traumatic joint disruption.

The viability of chondrocytes is significantly less along the edges of the fracture and on the articular surface than in other areas of cartilage. Importantly, a privileged area of viable cells is present in articular cartilage in the middle and deep zones away from the edge. This suggests that attempts to reconstruct traumatised joints are reasonable, as live tissue is present that might help heal the injury. The data presented also show distinct patterns of necrosis and apoptosis of chondrocytes following joint injury, particularly with respect to the time after injury. These differences in cell death are a significant step in understanding the effects of articular fracture.

No benefits in any form have been received or will be received from a commercial party related directly or indirectly to the subject of this article.

\section{References}

1. Brown TD, Johnston RC, Saltzman CL, Marsh JL, Buckwalter JA. Post-traumatic osteoarthritis: a first estimate of incidence, prevalence, and burden of disease. J Orthop Trauma 2006;20:739-44.

2. Murray MM, Zurakowski D, Vrahas MS. The death of articular chondrocytes after intra-articular fracture in humans. J Trauma 2004;56:128-31.

3. Kim HT, Lo MY, Pillarisetty R. Chondrocyte apoptosis following intraarticular fracture in humans. Osteoarthritis Cartilage 2002;10:747-9.

4. Hunziker EB, Michel M, Studer D. Ultrastructure of adult human articular cartilage matrix after cryotechnical processing. Microsc Res Tech 1997;37:271-84.

5. Eggli PS, Hunziker EB, Schenk RK. Quantitation of structural features characterizing weight- and less-weight-bearing regions in articular cartilage: a stereological analysis of medial femoral condyles in young adult rabbits. Anat Rec 1988;222:21727. 
6. Jeffery AK, Blunn GW, Archer CW, Bentley G. Three-dimensional collagen architecture in bovine articular cartilage. J Bone Joint Surg [Br] 1991;73-B:795-801.

7. Hwang WS, Li B, Jin LH, et al. Collagen fibril structure of normal, aging, and osteoarthritic cartilage. J Pathol 1992;167:425-33.

8. Milentijevic D, Helfet DL, Torzilli PA. Influence of stress magnitude on water loss and chondrocyte viability in impacted articular cartilage. J Biomech Eng 2003;125:594-601.

9. Milentijevic D, Torzilli PA. Influence of stress rate on water loss, matrix deformation and chondrocyte viability in impacted articular cartilage. J Biomech 2005;38:493502.

10. Torzilli PA, Grigiene R, Borrelli J Jr, Helfet DL. Effect of impact load on articular cartilage: cell metabolism and viability, and matrix water content. J Biomech Eng 1999;121:433-41.

11. Chen C-T, Burton-Wurster N, Borden C, et al. Chondrocyte necrosis and apoptosis in impact damaged articular cartilage. J Orthop Res 2001;19:703-11.

12. Klein TJ, Chaudhry M, Bae WC, Sah RL. Depth-dependent biomechanical and biochemical properties of fetal, newborn, and tissue-engineered articular cartilage. $J$ Biomech 2006;40:182-90.

13. Huang C-Y, Stankiewicz A, Ateshian GA, Mow VC. Anisotropy, inhomogeneity, and tension-compression nonlinearity of human glenohumeral cartilage in finite deformation. J Biomech 2005;38:799-809.

14. Guilak F. Compression-induced changes in the shape and volume of the chondrocyte nucleus. J Biomech 1995;28:1529-41.

15. Guilak F, Ratcliffe A, Mow VC. Chondrocyte deformation and local tissue strain in articular cartilage: a confocal microscopy study. J Orthop Res 1995;13:410-21.

16. Guilak F, Mow VC. The mechanical environment of the chondrocyte: a biphasic finite element model of cell-matrix interactions in articular cartilage. J Biomech 2000;33:1663-73

17. Eberhardt AW, Lewis JL, Keer LM. Normal contact of elastic spheres with two elastic layers as a model of joint articulation. J Biomech Eng 1991;113:410-17.

18. Ateshian GA, Wang H. A theoretical solution for the frictionless rolling contact of cylindrical biphasic articular cartilage layers. J Biomech 1995;28:1341-55.

19. Ewers BJ, Dvoracek-Driksna D, Orth MW, Haut RC. The extent of matrix damage and chondrocyte death in mechanically traumatized articular cartilage explants depends on rate of loading. J Orthop Res 2001;19:779-84.

20. Ewers BJ, Weaver BT, Haut RC. Impact orientation can significantly affect the outcome of a blunt impact to the rabbit patellofemoral joint. J Biomech 2002;35:1591-8.

21. Williamson AK, Chen AC, Masuda K, Thonar EJ, Sah RL. Tensile mechanical properties of bovine articular cartilage: variations with growth and relationships to collagen network components. J Orthop Res 2003;21:872.
22. Torzilli PA, Deng XH, Ramcharan M. Effect of compressive strain on cell viability in statistically loaded articular cartilage. Biomech Model Mechanobiol 2006:5:123-32.

23. Green DM, Noble PC, Bocell JR Jr, et al. Effect of early full weight-bearing after joint injury on inflammation and cartilage degradation. J Bone Joint Surg [Am] 2006;88-A:2201-9.

24. Donohue JM, Buss D, Oegema TR Jr, Thompson RC Jr. The effects of indirect blunt trauma on adult canine articular cartilage. J Bone Joint Surg [Am] 1983;65A:948-57.

25. Loening AM, James IE, Levenston ME, et al. Injurious mechanical compression of bovine articular cartilage induces chondrocyte apoptosis. Arch Biochem Biophys 2000;381:205-12.

26. Thompson RC Jr, Oegema TR Jr, Lewis JL, Wallace L. Osteoarthritic changes after acute transarticular load: an animal model. J Bone Joint Surg [Am] 1991;73A:990-1001.

27. Tew S, Kwan A, ann A, Thomson B, Archer C. The reactions of articular cartilage to experimental wounding: role of apoptosis. Arthritis Rheum 2000;43:215-25.

28. D'Lima DD, Hashimoto S, Chen PC, Colwell CW Jr, Lotz MK. Impact of mechanical trauma on matrix and cells. Clin Orthop 2001;391(Suppl):90-9.

29. Hooiveld M, Roosendaal G, Wenting M, et al. Short-term exposure of cartilage to blood results in chondrocyte apoptosis. Am J Pathol 2003;162:943-51.

30. Aizawa T, Kon T, Einhorn TA, Gerstenfeld LC. Induction of apoptosis in chondrocytes by tumor necrosis factor-alpha. J Orthop Res 2001;19:785-96.

31. Honkonen SE. Degenerative arthritis after tibial plateau fractures. J Orthop Trauma 1995;9:273-7.

32. Stevens DG, Beharry R, McKee MD, Waddell JP, Schemitsch EH. The longterm functional outcome of operatively treated tibial plateau fractures. J Orthop Trauma 2001;15:312-20

33. Volpin G, Dowd GS, Stein H, Bentley G. Degenerative arthritis after intra-articular fractures of the knee: long-term results. J Bone Joint Surg [Br] 1990;72-B:6348.

34. Buckwalter JA, Lohmander S. Operative treatment of osteoarthrosis: current practice and future development. J Bone Joint Surg [Am] 1994;76-A:1405-18.

35. Martin JA, Brown T, Heiner A, Buckwalter JA. Post-traumatic osteoarthritis: the role of accelerated chondrocyte senescence. Biorheology 2004;41:479-91.

36. Martin JA, Ellerbroek SM, Buckwalter JA. Age-related decline in chondrocyte response to insulin-like growth factor-l: the role of growth factor binding proteins. J Orthop Res 1997;15:491-8. 